\title{
QUANTUM MOLECULAR POLYHEDRA AND ATOMIC POPULATIONS
}

\author{
Ramon Carbó-Dorca \\ Secciò de Química Quàntica i Matemàtica, Centre Europeu de Recerca Teòrica, Universitat de Girona, 17071 Girona, \\ Catalonia, Spain.
}

\begin{abstract}
The present paper uses the LCAO MO theory formalism. The structure of the first order electronic density function is decomposed in two kinds of quantum polyhedra to discuss the behavior of quantum atomic populations. Among the many aspects one can consider about atomic populations here, the quantum mechanical structure of the density function is taken as the most important characteristic to think about. Apart of the usual one-electron basis set, centered in the molecular atoms, there is also discussed the possibility that the three-dimensional space where the molecular structures are described can be also the site of basis functions centered in points non-coincident with atomic positions.
\end{abstract}

Keywords: Density function; Quantum polyhedra; Mulliken atomic populations; Non-atomic function centers.

\section{INTRODUCTION}

Since the work of Mulliken ${ }^{1-4}$, first-order density is the standard tool to develop the concept of charge distribution in molecular structures from the quantum mechanical perspective. A more than a decade published paper ${ }^{5}$ which is a study about the chemical adequacy of Mulliken ${ }^{1}$ and Hirschfeld $^{6}$ atomic charges might be taken as another effort to elucidate some nuances of this subject. Atomic populations and charges of different origin and submitted to several computational schemes have been used in many aspects of theoretical chemistry. See for example an assorted set of references related with such a wide interest $^{7-15}$ along time. Atomic populations still deserve the attention of these quantum chemists like these of reference $^{5}$ who try to connect quantum and theoretical molecular information with the experimental chemistry lore.

One can start with the task to show that atomic populations and charges might be a way to associate the experimental ideas about molecular reactivity and behavior with computational chemistry. Thus, trying to inquire about the meaning of atomic populations constitute not a useless endeavor.

Some related work with the atomic populations by the present author might be useful to show the interest which has been present along with his research ${ }^{16-20}$.

In Mulliken's population description, one can feel he has considered the partition of the total number of molecular electrons, which is the sum of every atomic charge in a molecule when the LCAO MO wave function, and thus the density function has been calculated and known. In Mulliken's times, accurate wavefunctions for large molecular structures were not popular (in fact, they were barely available) enough as to test the result of his viewpoint on atomic populations, and thus he has described what he considered logical with the knowledge he could have at the moment of writing the series of papers $^{1-4}$ on this subject.This paper will try to provide more information on atomic populations, via the description of a dual set of quantum molecular polyhedra,

Auhtor for Correspondence: Ramon Carbó-Dorca, Secciò de Química Quàntica i Matemàtica, Centre Europeu de Recerca Teòrica, Universitat de Girona, 17071 Girona, Catalonia, Spain.

E-mail: ramoncarbodorca@gmail.com

Received: 10 Aug 2020; First Review: 29 Aug 2020; Second Review: 30 Aug 2020; Accepted: 1 Sep 2020.

Doi: https://doi.org/10.3126/sw.v14i14.34976 
which can be obtained from the knowledge of the density function. Once described such geometrical constructions, which can be associated with any molecular structure, some considerations about atomic populations will be finally supplied.

\section{QUANTUM MOLECULAR POLYHEDRA OVER MO'S}

Writing the density function as a superposition of squared modules of Molecular Orbitals (MO), as:

$\rho(\mathbf{r})=\sum_{I} \omega_{I}\left|\phi_{I}(\mathbf{r})\right|^{2}$

Where:

$$
\left\{\omega_{I} \mid I=1, N\right\} \wedge \sum_{I} \omega_{I}=N_{e l}
$$

are the occupation numbers of the MO. Here, they have been chosen with well-defined values in a set bearing an indefinite cardinality number $N$. They can be associated to integer values in the set $\forall \omega_{I} \in\{0,1,2\}$. Or more generally they can be considered a set of positive real numbers, however all the manipulated scalar numbers and function values are to be practically considered rational. Then, one can also suppose alternatively that: $\forall \boldsymbol{\omega}_{\mathrm{I}} \in \mathbb{Q}$

In any case the occupation numbers shall be submitted to the constraint (2).

The MO: $\mathrm{M}=\left\{\phi_{I}(\mathbf{r}) \mid I=1, N\right\}$ in turn can be expressed as linear combinations (LC) of an Atomic $^{1}$ Orbital (AO) basis set:

$\mathrm{X}=\left\{\chi_{\mu}(\mathbf{r}) \mid \mu=1, M\right\}$,

then each MO can be expressed in the usual way, as linear combinations of the chosen functions $\mathrm{X}$ :

${ }^{1}$ In modern times, since the massive introduction of Gaussian basis functions, the Atomic Orbitals (AO), which were employed to construct Molecular Orbitals (MO), have been substituted by optimized sets of functions. Here it is kept the old denomination name of $\mathrm{AO}$ but meaning a general description of atomic basis function sets. $\forall I: \phi_{I}(\mathbf{r})=\sum_{\mu} c_{\mu I} \chi_{\mu}(\mathbf{r})$

constituting the approach Mulliken called LCAO MO.

Now one can take the set of coefficients $\mathrm{C}=\left\{c_{\mu I} \mid \mu=1, M ; I=1, N\right\}$ as the coordinates of the

$\mathrm{MO}$ in $\mathrm{M}$ concerning the $\mathrm{AO}$ basis set $\mathrm{X}$.

It is interesting to represent the squares (of the modules if necessary) of the MO's, which can be written like considered real, but in fact, from the usual computational practice, all the numerically manipulated scalar numbers, like occupation numbers beforehand, and the function values are rational, then one can describe the positive definite functions derived from MO as:

$$
\begin{aligned}
\forall I:\left|\phi_{I}(\mathbf{r})\right|^{2} & =\sum_{\mu} \sum_{v} c_{\mu I} c_{v I} \chi_{\mu}(\mathbf{r}) \chi_{\nu}(\mathbf{r}) \\
& \equiv\left\langle\mathbf{c}_{I}|\mathbf{X}(\mathbf{r})| \mathbf{c}_{I}\right\rangle
\end{aligned}
$$

where $\left|\mathbf{c}_{I}\right\rangle$ and $\left\langle\mathbf{c}_{I}\right|$ are $N$-dimensional column and row vectors respectively, containing every MO coordinates with respect the AO basis set, while the $(M \times M)$ symmetric matrix $\mathbf{X}(\mathbf{r})$ is easily defined as:

$\mathbf{X}(\mathbf{r})=\left\{X_{\mu v}(\mathbf{r})=\chi_{\mu}(\mathbf{r}) \chi_{v}(\mathbf{r}) \mid \mu, v=1, M\right\}$.

Then, the density function might be easily rewritten in a compact form like:

$$
\begin{aligned}
\rho(\mathbf{r}) & =\sum_{I} \omega_{I}\left\langle\mathbf{c}_{I}|\mathbf{X}(\mathbf{r})| \mathbf{c}_{I}\right\rangle \\
& =\left\langle\left(\sum_{I} \omega_{I}\left|\mathbf{c}_{I}\right\rangle\left\langle\mathbf{c}_{I}\right|\right) * \mathbf{X}(\mathbf{r})\right\rangle=\langle\mathbf{D} * \mathbf{X}(\mathbf{r})\rangle
\end{aligned}
$$

Where the matrix Dcontains now the coordinates of the density function with respect of the elements of the matrix $\mathbf{X}(\mathbf{r})$, which acts in turn as a tensor function basis set. The expression (7) can be considered as a scalar product between both involved matrices. Just considering in expression (7) the product * as an inward one, see for more details reference ${ }^{21}$, and taking the sum of all the elements of the resultant matrix. A note about the matrix D is due now. The matrix $\mathbf{D}$ is usually named as the density matrix in the literature, some reflections on what 
may be considered the correct name for such a matrix were published not long ago $^{22}$ in an attempt to correct this misleading denomination.

Also, in several publications, see reference ${ }^{23}$ for instance, it has been discussed the possibility to consider the density function as a sum of MO bound densities, as one can write:

$\forall I: \mathbf{D}_{I}=\omega_{I}\left|\mathbf{c}_{I}\right\rangle\left\langle\mathbf{c}_{I}\right| \rightarrow \mathbf{D}=\sum_{I} \mathbf{D}_{I}$

and then define the MO density functions as:

$\forall I: \rho_{I}(\mathbf{r})=\left\langle\left(\omega_{I}\left|\mathbf{c}_{I}\right\rangle\left\langle\mathbf{c}_{I}\right|\right) * \mathbf{X}(\mathbf{r})\right\rangle=\left\langle\mathbf{D}_{I} * \mathbf{X}(\mathbf{r})\right\rangle$

therefore, it is simple to see that:

$\rho(\mathbf{r})=\sum_{I} \rho_{I}(\mathbf{r})$

The set of MO density functions:

$\mathrm{P}=\left\{\rho_{I}(\mathbf{r}) \mid I=1, N\right\}$

can be considered as a quantum polyhedron, see references $^{24-33}$ for more details. Every MO density function acts as a vertex of the quantum polyhedron $\mathrm{P}$. The Minkowski norms of the vertices of the quantum polyhedron (11), produce the occupation number of the attached MO:

$\forall I:\left\langle\rho_{I}\right\rangle=\int_{D} \rho_{I}(\mathbf{r}) \mathrm{d} \mathbf{r}=\omega_{I}$

and the sum of the Minkowski norms yield the number of molecular electrons as shown in equation (2), according to the Minkowski norm of the molecular electronic density:

$\langle\rho\rangle=\int_{D} \rho(\mathbf{r}) \mathrm{d} \mathbf{r}=N_{e l}$

which also yields the number of electrons $N_{e l}$.

Such an obvious manipulation of the first-order density function in an LCAO MO framework permits to obtain information from the quantum environment of any molecular structure. In some manner, the quantum polyhedron framework associated with the first-order density function has been described in previous papers ${ }^{24-33}$. Although it might be interesting to use a similar formalism to study atomic populations.

\section{QUANTUM MOLECULAR POLYHEDRA OVER ATOMIC CENTERS}

Additionally, one can define another partition of the density function in terms of atomic densities. The AO basis set is constructed by the union of function subsets, belonging to an atom entering the molecular structure (or a point in three-dimensional Cartesian space suitably chosen in the molecular surroundings, not necessarily coincident with an atomic site) whose density function has to be computed.

The basis set $\mathrm{X}$ defined in the equation (3), considering the centers where the $\mathrm{AO}$ are associated, can be rewritten in this way:

$$
\begin{aligned}
\mathrm{X}=\bigcup_{K} \mathrm{X}_{K} & \wedge \\
\mathrm{X}_{K} & =\left\{\chi_{\mu}^{K}(\mathbf{r}) \mid \mu=1, M_{K} ; K=1, C\right\}
\end{aligned}
$$

Where the index $K$ runs over the number of centers chosen, $C$, where the AO functions are placed, and $M_{K}$ is the number of functions settled at each center $\mathrm{K}$. Therefore, the matrix $\mathbf{X}(\mathbf{r})$ can be expressed over the submatrices associated to the $\mathrm{AO}$ centers as:

$\mathbf{X}(\mathbf{r})=\left\{\mathbf{X}_{K L}(\mathbf{r}) \mid K, L=1, C\right\}$,

a similar partition of the matrix of the coordinates of the density matrix can be also performed, so it can be written:

$$
\begin{aligned}
\forall K: \mathbf{D}_{I}^{K L} & =\left|\mathbf{c}_{I}^{K}\right\rangle\left\langle\mathbf{c}_{I}^{L}\right| \\
& \leftarrow\left\langle\mathbf{c}_{I}^{L}\right|=\left\{c_{\mu I}^{L} \mid \mu=1, M_{L} ; I=1, N ; L=1, C\right\}
\end{aligned}
$$

and in this manner one can construct another quantum polyhedron, which will be structured by vertices made via partial density functions associated with each center, which can be written in turn as:

$$
\begin{aligned}
\forall K: \rho^{K}(\mathbf{r}) & =\sum_{L}\left\langle\left(\sum_{I} \omega_{I}\left|\mathbf{c}_{I}^{K}\right\rangle\left\langle\mathbf{c}_{I}^{L}\right|\right) * \mathbf{X}^{K L}(\mathbf{r})\right\rangle \\
& =\sum_{L}\left\langle\mathbf{D}^{K L} * \mathbf{X}^{K L}(\mathbf{r})\right\rangle
\end{aligned}
$$


with the final result allowing to express the density function alternatively as:

$\rho(\mathbf{r})=\sum_{K} \rho^{K}(\mathbf{r})^{\cdot}$

One can see now that the set of density functions, centered at each position around the molecule, also corresponds to an alternative quantum polyhedron vertex set:

$$
\mathrm{Q}=\left\{\rho^{K}(\mathbf{r}) \mid K=1, C\right\}
$$

The vertices of the quantum polyhedron (19) might be used to produce the atomic populations (one can admit the possibility that basis set function centers could be placed somewhere in the 3-dimensional space around the molecule, so one can talk about center populations).

Atomic or center populations are computed using their Minkowski norms:

$$
\forall K: Q_{K}=\left\langle\rho^{K}\right\rangle=\int_{D} \rho^{K}(\mathbf{r}) \mathrm{d} \mathbf{r}
$$

forming a scalar set:

$Q=\left\{Q_{K} \mid K=1, C\right\} \rightarrow \sum_{K} Q_{K}=N_{e l}$

whose sum is the number of electrons $N_{e l}$, in accord with the property of the Minkowski norm of the density function as discussed beforehand in the equation (13).

\section{OUT-OF-ATOM MULTICENTER POPULATIONS}

An interpretative problem appears when centers which do not coincide with molecular atoms are used to locate basis set functions ${ }^{2}$. Then some of the calculated populations' will bear, as a counterpart of the atomic centers, some fraction of the number of molecular electrons, which will be present whenever at some center A there are located some functions, say: $\left\{\chi_{\alpha}^{A} \mid \alpha=1, M_{A}\right\}$ of the basis set. In this manner giving rise to a vertex of the quantum

${ }^{2}$ This is an option the usual quantum chemistry programs apparently ignore, or it is included as an option but the users don't choose this possibility anymore. Such forgotten feature can explain why it seems that the problem, associated to this out-of-atoms centering of basis set functions, doesn't exist. polyhedron: $\rho^{A}(\mathbf{r})$, and therefore to a non-null center population: $Q_{A}=\left\langle\rho^{A}\right\rangle$.

This possible occurrence seems counterintuitive, using the wording employed in reference ${ }^{5}$. Moreover: which interpretation can be associated with such an electron fractionation outside the atomic molecular centers?

Except in some exceptional cases, where the extra-atomic center is chosen for some experimental connected purpose, it seems that not a plausible interpretation can be established to out-of-atom populations, except in describing lone pair regions or similar electronic distributions, if any.

The reason can be found in the space structure, where molecules are located. Note also that the number of nonatomic centers, where basis functions can be placed, might be arbitrarily large. Thus, the molecular electrons will appear fractionated arbitrarily, depending on the extra centers used.

Moreover, the nature of the functional Hilbert space where the basis set functions are described is also flexible enough to admit freedom about the functions to use. There is an infinite number of functions that can be chosen as building blocks to construct $\mathrm{MO}$, and therefore the molecular polyelectronic wave function.

To grasp this possibility one can peruse the papers ${ }^{34-39}$ on Gaussian space enfolding, where a function, in the most simple of cases, is centered at all the points of some chosen space, which although arbitrary in structure, it can be the usual three-dimensional Cartesian space holding the atomic positions of the molecular structures.

One can imagine that there could potentially be an infinite set of vertices in the quantum molecular polyhedron(19), as the number of centers underwent a trend like: $C \rightarrow \infty$ in a space enfolding. Thus, one can suppose that the population set can be seen in the same way and that the scalar population set defined in (21) might be composed in this case by an infinite number of elements. Such a new structure of the populations one can envisage forms an 
infinite-dimensional vector that could be transformed into the property:

$$
Q_{\infty}=\{Q(K) \mid K \in \mathbb{Q}\} \rightarrow \underline{\int} D Q(K) d k=N_{e l}
$$

\section{BASIS SET UNBALANCE AND HYDROGEN ATOM POPULATIONS}

Such a molecular environment space, the feature out-ofatom centers, has not been discussed in the paper ${ }^{5}$, nor as far as the present author knows in many other papers. The signers of reference ${ }^{5}$ conclude, however, that in Mulliken treatment it is present a systematic behavior of the Hydrogen populations not conforming to the chemical intuition. It is nice to have noted such a feature because it might be perhaps easily explained.

Even in large basis sets, which are featured in HF, DFT or whatever procedure which is used to obtain occupation numbers and MO's, while heavy atoms are associated to a large number of basis set functions, the number of functions attached to Hydrogen atoms is systematically kept lower than the rest, much less than Carbon, Nitrogen or Oxygen, for instance, to do not speak of Bromine or Iodine or heavier atoms. This can be summarized by the relation of the function numbers: $M_{H} \ll<M_{\text {heavy metals }}$.

The unbalanced number of functions creates unbalanced vertices of the quantum polyhedron (19), constructed as in equation (17). This could be the origin of this characteristic behavior of Hydrogen Mulliken populations, and possibly it can be found among other population definitions. Albeit the authors of reference ${ }^{5}$ indicate that the Hydrogen charges are stable along with changes in basis sets. The stability found $i^{5}$ with changing basis sets could be attributed to the fact that the unbalance between Hydrogen and heavier atoms continues to be similar or constant, whenever the basis set changes. The Hydrogen number of functions also has an incidence in the absolute value of the coefficients of each MO connected with these atoms, and this produces at the end, a set of not so rational (from chemistry lore) values of their populations.
Moreover, placing basis functions out from the atomic centers not only provides a possible counterintuitive fractioning of the molecular electrons but proves that Hilbert space geometry and chemistry could be paradoxically unconnected. Indeed, locating an extended number of functions in the molecular space environment will certainly produce a better wave function from the variational theorem perspective, but will possibly yield awful electronic population sets as defined in (21) or (22).

On the other hand, the out-of-atoms basis set location might even prevent or reduce the incidence of quasisingularity, or computational singularity of the overlap matrix $\mathbf{S}$ associated to a chosen basis set, which can be obtained by integrating the matrix of the equation (15):

$\forall K, L: \mathbf{S}_{K L}=\int_{D} \mathbf{X}_{K L}(\mathbf{r}) \mathrm{d} \mathbf{r} \rightarrow \mathbf{S}=\left\{\mathbf{S}_{K L} \mid K, L=1, C\right\}$

The quasi-singularity of the overlap matrix appears when the number of basis set functions centered in atomic sites grows because the total number of atomic centered functions also obviously grows, and some functions become linearly dependent at the computational precision used. This computational drawback shows in the form of the numerical appearance of very small overlap matrix eigenvalues, whose absolute values due to the limited machine precision are almost null or null. This provokes the ill-definition of the overlap matrix inverse, because in this case there might appear a quasi-non-number error feature in the inverse computation process. It seems that this drawback has been arranged with the aid of numerical recipes. Albeit, in doing so, the problem doesn't disappear and can provoke computational errors, perhaps of some importance, in the posterior computational treatment in search of the MO.

\section{PROMOLECULAR DENSITY FUNCTIONS}

Another problem, which the possibility of using out-ofatomic centers functions might put in evidence, will certainly appear in the construction of the so-called promolecular density functions. Promolecular densities can be constructed as a superposition of atomic densities, 
built with the same or taking simplified basis set functions, in any case considering the attached molecular atom isolated. For more information about promolecular densities and its varied uses, see references ${ }^{40-47}$, for example.

One can suppose for a given molecule exists a set of atomic densities, similar to the ones of the quantum polyhedron (19), but bearing no interaction whatsoever with the rest of the molecular basis set. Let's define such possible approximate quantum polyhedra as:

$$
\mathrm{A}=\left\{p_{I}(\mathbf{r}) \mid I=1, A\right\}
$$

where $A$ is the number of atoms of the molecule studied. Then the promolecular density is constructed like:

$p(\mathbf{r})=\sum_{I} p_{I}(\mathbf{r})$

The atomic promolecular functions are Minkowski normalized to the atomic charge of the nuclei:

$\forall I:\left\langle p_{I}(\mathbf{r})\right\rangle=\int_{D} p_{I}(\mathbf{r}) \mathrm{d} \mathbf{r}=Z_{I}$,

Thus, the promolecular density functions possess a Minkowski norm which is the same as any molecular density function:

$\langle p(\mathbf{r})\rangle=\int_{D} p(\mathbf{r}) \mathrm{d} \mathbf{r}=\sum_{I}\left\langle p_{I}(\mathbf{r})\right\rangle=\sum_{I} Z_{I}=N_{e l}$.

The definitions (24) and (25) do not contain any promolecular atomic density centered in a non-atomic position. Hence, a comparison of the kind that is used in the so-called Hirschfeld charges ${ }^{6}$ cannot be done when the density function possesses centers, which are not located at the molecular atoms.

This is another drawback, which affects this time to the calculation of Hirschfeld atomic charges, when additional non-atom centered sets of functions are used in the main computation of MO's.

\section{CONCLUSIONS}

The authors of reference ${ }^{5}$ in their conclusions have focused their analysis on the Hydrogen populations, which is an interesting subject indeed. Apparently, no attempt is made from their part to deepen on the mathematical structure lying in the definition of the density function, except for some considerations to ameliorate the results of the original descriptions. Thus, the present paper can be considered as an addition to their particular findings but having a slightly more general purpose.

Showing, perhaps, that the so-called atomic populations, hence the atomic charges, are somehow arbitrarily constructed, unless one uses a better background within a more correct quantum mechanical point of view, as the choice of an operator as it has been tried to show in the paper of reference ${ }^{17}$. There it is shown that Mulliken's atomic populations can be considered as expectation values of some well-defined operator. If these population values are not coincident with chemical intuition might be because perhaps the underlying basis set is not welldefined to adapt to chemical lore experimental results but is well suited to provide with a correct variational electronic energy.

Conceivably, the manipulation of the vertices of the quantum molecular polyhedra, which has been largely described and used, see references ${ }^{24-33}$, might be considered as an additional general-purpose tool to interpret wavefunctions for chemical use instead of generally centered populations.

\section{COMPLIANCE WITH ETHICAL STANDARDS}

Conflict of interest: The author state that there is no conflict of interest related to this work.

\section{ACKNOWLEDGEMENT}

The author is indebted to Prof. Dr. Vinay Kumar Jha, Central Department of Chemistry, Tribhuvan University, for his help in building up the present manuscript.

\section{REFERENCES}

1. Mulliken, R. S. 1955. Electron Population Analysis on LCAO-MO Molecular Wave Functions I. Journal of Chemical Physics. 23 $1833-1840$

2. Mulliken, R. S. 1955. Electron Population Analysis on LCAO-MO Molecular Wave Functions II. Overlap Populations, Bond Orders, 
and Covalent Bond Energies. Journal of Chemical Physics. 23: 1841-1846.

3. Mulliken, R. S. 1955. Electron Population Analysis on LCAO-MO Molecular Wave Functions III. Effects of Hybridization on Overlap and Gross AO Populations. Journal of Chemical Physics. 23: 23382342 .

4. Mulliken, R. S. 1955. Electron Population Analysis on LCAO-MO Molecular Wave Functions IV. Bonding and Antibonding in LCAO and Valence-Bond Theories". Journal of Chemical Physics. 23: 2343-2346.

5. Saha, S., Roy, R. K. and Ayers P. W. 2009. Are the Hirshfeld and Mulliken Population Analysis Schemes Consistent with Chemical Intuition? International Journal of Quantum Chemistry. 109: 17901806.

6. Hirshfeld, F. L. 1977. Bonded-atom Fragments for Describing Molecular Charge Densities. Theoretica Chimica. Acta. 44: 129138.

7. Cioslowski, J. 1989. A New Population Analysis Based on Atomic Polar Tensors. Journal of American Chemical Society. 111: 83338336.

8. Huzinaga, S., Sakai, Y., Miyoshi, E. and Narita S. 1990. Extended Mulliken electron population analysis. The Journal of Chemical Physics. 93: 3319-3325.

9. de Oliveira, A. E., Guadagnini, P. H. Haiduke, R. L. A. and Bruns R. E. 1999. A Simple Potential Model Criterion for the Quality of Atomic Charges. Journal of Physical Chemistry. A 103: 4918-4924.

10. Bultinck, P., Langenaeker, W., Lahorte, P., De Proft, F., Geerlings, P. Alsenoy, C. Van and Tollenaere, J. P. 2002. The Electronegativity Equalization Method II: Applicability of Different Atomic Charge Schemes. Journal of Physical Chemistry. A-106: (2002) 7895-7901.

11. Kang, Y. K. and Scheraga, H. A. 2008. An Efficient Method for Calculating Atomic Charges of Peptides and Proteins from Electronic Populations. Journal of Physical Chemistry B- 112: 5470-5478.

12. T. Verstraelen, T., Pauwels, E., De Proft, F., Van Speybroeck, V., Geerlings, P. and Waroquier, M. 2012. Assessment of Atomic Charge Models for Gas-Phase Computations on Polypeptides. Journal of Chemical Theory and Computation. 8: 661-676.

13. Ionescu, C.-M., Geidl, S., Vařeková, R. S. and Koča, J. 2013. Rapid Calculation of Accurate Atomic Charges for Proteins via the Electronegativity Equalization Method. Journal of Chemical Information and Modeling. 53: 2548-2558.

14. Penchoff, D. A., Peterson, Ch. C., Quint, M. S., Auxier II, J. D., Schweitzer, G. K., Jenkins, D. M., Harrison, R. J. and Hall, H. L. 2018. Structural Characteristics, Population Analysis, and Binding Energies of $\left[\mathrm{An}\left(\mathrm{NO}_{3}\right)\right]^{2+}$ (with $\mathrm{An}=\mathrm{Ac}$ to $\mathrm{Lr}$ ). ACS Omega. 3: 14127-14143.

15. Nikolova, V., Cheshmedzhieva, D., Ilieva, S. and Galabov, B. 2019. Atomic Charges in Describing Properties of Aromatic Molecules. Journal of Organic Chemistry. 84: 1908-1915.
16. Carbó-Dorca, R. and Arnau, C. 1978. A general representation of atomic orbital charge distributions with some applications to Mulliken's approximation and population analysis. Gazzetta Chimica Italiana. 108: 171-176.

17. Carbó-Dorca, R. and Bultinck, P. 2004. Quantum mechanical basis for Mulliken population analysis. Journal of Mathematical Chemistry. 36: 231-239.

18. R. Carbó-Dorca, R. 2010. LCAO MO First Order Density Functions: Partition in Monocentric and Bicentric Terms, Reciprocal MO Spaces, Invariant Transformations and Atomic Populations (special issue on conceptual DFT). Journal of Molecular Structure (THEOCHEM). 943: 32-41.

19. Carbó-Dorca, R. 2013. Simple Comparison of Atomic Population and Shape Atomic Populations Distributions between two molecular structures with a coherent number of atoms. Journal of Mathematical Chemistry. 51: 774-784.

20. Carbó-Dorca, R. 2018. Atomic thermal voltage population distributions. Journal of Mathematical Chemistry. 56: 1357-1359.

21. Carbó-Dorca, R. 2000. Quantum quantitative structure-activity relationships (QQSAR): A comprehensive discussion based on inward matrix products, employed as a tool to find approximate solutions of strictly positive linear systems and providing a QSAR-Quantum similarity measures connection. Proceedings of European Congress on Computational Methods in Applied Sciences and Engineering. ECCOMAS 2000. Barcelona, 11-14 September 2000 ISBN-84-89925-70-4, 1-31.

22. Carbó-Dorca, R. 2013. On Density Function Coordinate Matrix”. Journal of Mathematical Chemistry. 51: 1681-1683.

23. R. Carbó-Dorca, R. 2005. Mathematical Elements of Quantum Electronic Density Functions. Advances in Quantum Chemistry. 49: 121-207

24. R. Carbó-Dorca, R. 2014. Multimolecular Polyhedra and QSPR. Journal of Mathematical Chemistry. 52: 1848-1856.

25. R. Carbó-Dorca, R. 2015. Quantum Polyhedra, Definitions, Statistics and the Construction of a Collective Quantum Similarity Index. Journal of Mathematical Chemistry. 53: 171-182.

26. Carbó-Dorca, R. and Barragán, D. 2015. Communications on Quantum Similarity (4): Collective distances computed by means of Similarity Matrices, as generators of intrinsic ordering among Quantum Multimolecular Polyhedra. WIRES Computational Molecular Science. 5: 380-404.

27. Carbó-Dorca, R. 2015. An Isometric Representation Problem Related with Quantum Multimolecular Polyhedra and Similarity. Journal of Mathematical Chemistry. 53: 1750-1758.

28. Carbó-Dorca, R. 2015. “An Isometric Representation Problem in Quantum Multimolecular Polyhedra and Similarity:(2) Synisometry. Journal of Mathematical Chemistry. 53: 1867-1884.

29. Carbó-Dorca, R. 2016. Quantum Molecular Polyhedra in LCAOMO Theory. Molecular Physics. 114 (7-8): 1236-1249. 
30. Carbó-Dorca, R. 2016. Aromaticity, quantum molecular polyhedra and quantum QSPR. Journal of Computational Chemistry. 37: 7882.

31. Carbó-Dorca, R. and González, S. 2016. Notes in QSPR (4): Quantum Multimolecular Polyhedra, Collective Vectors, Quantum Similarity and Quantum QSPR fundamental equation. Management Studies. 4: 33-47.

32. Carbó-Dorca, R. 2016. A Study on the Centroid Vector of a Polyhedron. Journal of Mathematical Chemistry. 54: 51-71.

33. Carbó-Dorca, R. 2017. A Theorem on the Gram matrix of a Polyhedron. Journal of Mathematical Chemistry. 55: 79-97.

34. Besalú, E. and Carbó-Dorca, R. 2011. N-dimensional Euclidian Space enfoldment. Journal of Mathematical Chemistry. 49: 22312243.

35. Carbó-Dorca, R. and Besalú, E. 2011. "Geometry of $N$-dimensional Euclidian Space enfoldments. Journal of Mathematical Chemistry. 49: 2244-2249.

36. Carbó-Dorca, R. 2013. Enfolded conformational spaces: definition of the chemical quantum mechanical multiverse under BornOppenheimer approximation. Journal of Mathematical Chemistry. 51: 1092-1098.

37. Carbó-Dorca, R. 2013. Position-momentum Heisenberg uncertainty in Gaussian enfoldments of Euclidian space. Journal of Mathematical Chemistry. 51: 420-426.

38. Carbó-Dorca, R. 2016. Time Vectors and Particle Swarms Defined as Polyhedra in Spherically Enfolded Spaces. Journal of Mathematical Chemistry. 54: 1751-1757.

39. Chang, J. and Carbó-Dorca R. 2020. A Quantum Similarity Discussion about Einstein-Podolsky-Rosen (EPR) Paradox in Gaussian Enfolded Spaces. Journal of Mathematical Chemistry, (Accepted June 27, 2020).

40. Constans, P., Fradera, X., Amat, L. and Carbó-Dorca R. 1996. Quantum Molecular Similarity Measures (QMSM) and the Atomic Shell Approximation (ASA). Proceedings of the 2nd. Girona Seminar on Molecular Similarity JAI PRESS INC. Greenwich (Conn.). July 1995. Advances in Molecular Similarity. 1: 187-211.

41. Gironés, X., Amat, L. and Carbó-Dorca, R. 1998. A comparative study of Isodensity surfaces using ab initio and ASA density functions. Journal of Molecular Graphics \& Modeling. 16: 190196.

42. Carbó-Dorca, R. 1998. On the statistical interpretation of Density Functions: ASA, Convex Sets, discrete Quantum Chemical molecular representations, Diagonal Vector Spaces and related problems. Journal of Mathematical Chemistry. 23: 365-375.

43. Carbó-Dorca, R. 1998. Fuzzy sets and Boolean tagged sets, vector semispaces and convex sets, QSM and ASA density functions, diagonal vector spaces and quantum Chemistry. JAI PRESS INC. Greenwich (Conn.)., Advances in Molecular Similarity. 2: 43-72.

44. Amat, L. and Carbó-Dorca, R. 2000. Molecular Electronic Density Fitting Using Elementary Jacoby Rotations under Atomic Shell Approximation (ASA). Journal of Chemical Information and Computer Sciences. 40: 1188-1198.

45. Gironés, X., Carbó-Dorca, and Mezey R. P. G. 2001. Application of Promolecular ASA Densities to Graphical Representation of Density Functions of Macromolecular Systems. Journal of Molecular Graphics and Modelling. 19: 343-348.

46. Amat, L1. And Carbó-Dorca R. 2002. Use of promolecular ASA density functions as a general algorithm to obtain starting MO in SCF calculations. International Journal of Quantum Chemistry. 87: 59-67.

47. Carbó-Dorca, R. and Besalú, E. 2012. On the nature of Atomic Shell Approximation (ASA) Electrostatic Molecular Potentials (EMP). Journal of Mathematical Chemistry. 50: 981-988. 